\title{
Quantum SUSY Algebra of $Q$-lumps in the Massive Grassmannian Sigma Model
}

\author{
Hiroaki Nakajima*, Phillial $\mathrm{Oh}^{\dagger}$, and Sunyoung Shin ${ }^{\ddagger}$ \\ Department of Physics and Institute of Basic Science, \\ Sungkyunkwan University, Suwon 440-746 Korea
}

(Dated: October 29, 2018)

\begin{abstract}
We compute the $\mathcal{N}=2$ SUSY algebra of the massive Grassmannian sigma model in $2+1$ dimensions. We first rederive the action of the model by using the Scherk-Schwarz dimensional reduction from $\mathcal{N}=1$ theory in $3+1$ dimensions. Then, we perform the canonical quantization by using the Dirac method. We find that a particular choice of the operator ordering yields the quantum SUSY algebra of the $Q$-lumps with cental extension.
\end{abstract}

PACS numbers: 11.30.Pb, 03.70+k, 11.10.-z

Keywords: $Q$-lump, supersymmetric algebra, BPS, Grassmannian model

\section{INTRODUCTION}

$Q$-lumps [1, 2] are time dependent topological objects which are stabilized by a Noether charge of global symmetry like $Q$-balls [3] as well as a topological charge. It is well-known that $Q$-lumps are BPS objects and preserve a fraction of supersymmetries. In this respect, there has been a great deal of interest in this object. In particular, the $Q$-lump solutions in massive sigma model are investigated in [4, 5, 6, 7, 8, 9], and the relation to the D-brane configuration is also studied in [10, 11, 12, 13]. In relation with supersymmetric gauge theories, the massive sigma model can be realized as an effective action of non-Abelian vortex strings, which have been discovered recently [14, 15, 16]. $Q$-lumps in supersymmetric gauge theories are examined in [17].

According to the well-known result of [18, 19], the SUSY algebra of the $Q$-lumps will include the central charges. In relation with nonlinear sigma model, the central charges of the $\mathrm{CP}^{N}$ model were computed at the classical level [20, 21]. In this paper, we explicitly compute the quantum SUSY algebra of $Q$-lumps in the massive Grassmannian model. The resulting SUSY algebra can be expected to change by a mass term in the Hamiltonian compared with [20, 21], but the precise expressions of the central charges and the Hamiltonian depend on the operator ordering and we find that enforcing the SUSY algebra with central charges is closely tied with some particular ordering prescription (See eqs. (III.24), (IV.12), (IV.13) ).

One way of obtaining the SUSY algebra is deriving it from supersymmetric transformation rules. However, this passage usually cannot deal with the operator ordering problem. Instead, we perform the canonical quantization via the Dirac method by carefully taking into account the ordering ambiguity. It turns out that a specific choice of ordering yields the SUSY algebra with central extension.

We first derive the off-shell action of the massive Grassmannian sigma model via Scherk-Schwarz dimensional reduction [22], then the Dirac analysis of constraints is applied to get classical SUSY algebra. After that we quantize the SUSY algebra by considering the ordering problem.

\section{II. $\mathcal{N}=2$ OFF-SHELL SUPERSYMMETRIC GRASSMANNIAN ACTION}

We consider the Grassmannian sigma model of which the target space is the coset space $S U(N+M) / S(U(N) \times$ $U(M)) .{ }^{1}$ It is possible to obtain $\mathcal{N}=2$ supersymmetric action in $2+1$ dimensions using superfield formalism by dimensional reduction from $\mathcal{N}=1$ supersymmetric model in $3+1$ dimensions. The chiral and antichiral fields in the $(N+M) \times M$ matrix and the vector fields in the $M \times M$ matrix in 3+1 dimensions are defined as follows

$$
\begin{array}{rlrl}
\Phi\left(x_{L}, \theta\right) & =\phi\left(x_{L}\right)+\sqrt{2} \theta \psi\left(x_{L}\right)+\theta \theta F\left(x_{L}\right), & & x_{L}^{\mu}=x^{\mu}-i \theta \sigma^{\mu} \bar{\theta}, \\
\bar{\Phi}\left(x_{R}, \bar{\theta}\right) & =\phi\left(x_{R}\right)+\sqrt{2} \bar{\theta} \bar{\psi}\left(x_{R}\right)+\bar{\theta} \bar{\theta} \bar{F}\left(x_{R}\right), & & x_{R}^{\mu}=x^{\mu}+i \theta \sigma^{\mu} \bar{\theta}, \\
V & =2 \bar{\theta} \bar{\sigma}^{\mu} \theta A_{\mu}+i(\theta \theta)(\bar{\theta} \bar{\lambda})-i(\bar{\theta} \bar{\theta})(\theta \lambda)+(\theta \theta)(\bar{\theta} \bar{\theta}) \tau,
\end{array}
$$

\footnotetext{
* E-mail address: nakajima@skku.edu

$\dagger$ E-mail address: ploh@skku.edu

¥ E-mail address: sihnsy@skku.edu

${ }^{1}$ Extended supersymmetries of massive nonlinear sigma models have been studied in various dimensions 24, 25, 26, 27, 28, 29, 30, 31, 32].
} 
and the Lagrangian can be written in the form [20, 23]

$$
\int d^{4} \theta \operatorname{tr}\left[\bar{\Phi} \Phi e^{V}-V\right]=\operatorname{tr}\left[\overline{D_{\mu} \phi} D^{\mu} \phi+\frac{i}{2}\left(-\overline{D_{\mu} \psi} \bar{\sigma}^{\mu} \psi+\bar{\psi} \bar{\sigma}^{\mu} D_{\mu} \psi\right)+\bar{F} F-\frac{i}{\sqrt{2}} \bar{\psi} \phi \bar{\lambda}+\frac{i}{\sqrt{2}} \bar{\phi} \psi \lambda+\tau(\bar{\phi} \phi-1)\right]
$$

In order to obtain massive model in $2+1$ dimensions we apply the Scherk-Schwarz dimensional reduction [22, 24] specifying that the fields in the $x^{3}$-direction are moving along orbits of the Killing vectors $f(\phi)$ and $\bar{f}(\bar{\phi})$ in the Grassmannian manifold

$$
\frac{\partial \phi}{\partial x^{3}}=f(\phi), \quad \frac{\partial \bar{\phi}}{\partial x^{3}}=\bar{f}(\bar{\phi}), \quad \frac{\partial \psi}{\partial x^{3}}=\partial f(\phi) \psi, \quad \frac{\partial \bar{\psi}}{\partial x^{3}}=\bar{\partial} \bar{f}(\bar{\phi}) \bar{\psi}
$$

The general forms of the Killing vector $f(\phi)$ and $\bar{f}(\bar{\phi})$ are given by

$$
f(\phi)=i \mathcal{M} \phi, \quad \bar{f}(\bar{\phi})=-i \bar{\phi} \mathcal{M}
$$

because the isometry $S U(N+M)$ is linearly realized and the matrix $\mathcal{M}$ is diagonal element of it ${ }^{2}$. We substitute (II.5) and (II.6) into (II.4) to obtain (with $A_{3} \equiv \sigma$ )

$$
\begin{aligned}
\mathcal{S}=\int d^{3} x \operatorname{tr} & {\left[\left|D_{\mu} \phi\right|^{2}+\frac{i}{2}\left(-\overline{D_{\mu} \psi} \gamma^{\mu} \psi+\bar{\psi} \gamma^{\mu} D_{\mu} \psi\right)+\bar{F} F-\bar{\phi} \mathcal{M}^{2} \phi+2 \bar{\phi} \mathcal{M} \phi \sigma-\bar{\phi} \phi \sigma^{2}+\bar{\psi} \mathcal{M} \psi-\bar{\psi} \psi \sigma\right.} \\
& \left.-\frac{i}{\sqrt{2}} \bar{\psi} \phi \bar{\lambda}+\frac{i}{\sqrt{2}} \bar{\phi} \psi \lambda+\tau(\bar{\phi} \phi-1)\right]
\end{aligned}
$$

By constraints of the system

$$
\bar{\phi} \phi=I, \quad \bar{\phi} \psi_{\alpha}=0=\bar{\psi}_{\alpha} \phi
$$

and eliminating auxiliary fields

$$
\begin{aligned}
F & =0, & \bar{F} & =0, \\
\sigma & =\frac{1}{2}(2 \bar{\phi} \mathcal{M} \phi-\bar{\psi} \psi), & A^{\mu} & =\frac{1}{2}\left(i \partial^{\mu} \bar{\phi} \phi-i \bar{\phi} \partial^{\mu} \phi-\bar{\psi} \gamma^{\mu} \psi\right) .
\end{aligned}
$$

we get the action

$$
\begin{aligned}
\mathcal{S}=\int d^{3} x \operatorname{tr} & {\left[\left|\partial_{\mu} \phi\right|^{2}+\frac{i}{2}\left(-\partial_{\mu} \bar{\psi} \gamma^{\mu} \psi+\bar{\psi} \gamma^{\mu} \partial_{\mu} \psi\right)-\frac{1}{4}\left(i \partial_{\mu} \bar{\phi} \phi-i \bar{\phi} \partial_{\mu} \phi-\bar{\psi} \gamma_{\mu} \psi\right)^{2}\right.} \\
+ & \left.\left(\bar{\phi} \mathcal{M} \phi-\frac{1}{2} \bar{\psi} \psi\right)^{2}-\bar{\phi} \mathcal{M}^{2} \phi+\bar{\psi} \mathcal{M} \psi\right]
\end{aligned}
$$

With the definition of $\mathcal{M}=m \mathcal{P}$ where $\mathcal{P}$ is the $(N+M) \times(N+M)$ Hermitian projection matrix satisfying $\mathcal{P}^{2}=\mathcal{P}$ and $m$ is a real positive number, the action is

$$
\begin{aligned}
& \mathcal{S}=\int d^{3} x \operatorname{tr} {\left[\left|\partial_{\mu} \phi\right|^{2}+\frac{i}{2}\left(-\partial_{\mu} \bar{\psi} \gamma^{\mu} \psi+\bar{\psi} \gamma^{\mu} \partial_{\mu} \psi\right)-\frac{1}{4}\left(i \partial_{\mu} \bar{\phi} \phi-i \bar{\phi} \partial_{\mu} \phi-\bar{\psi} \gamma_{\mu} \psi\right)^{2}\right.} \\
&\left.+\left(m \bar{\phi} \mathcal{P} \phi-\frac{1}{2} \bar{\psi} \psi\right)^{2}-m^{2} \bar{\phi} \mathcal{P} \phi+m \bar{\psi} \mathcal{P} \psi\right]
\end{aligned}
$$

which is the same as the one given in [9].

\section{DIRAC ANALYSIS}

In this section, we perform the Dirac analysis, which is useful to calculate the algebra of constrained system, to obtain the SUSY algebra of the action (II.12). The massless supersymmetric $\mathrm{CP}^{N}$ model was studied in [20, 21]. The

\footnotetext{
${ }^{2}$ However, the traceless condition for $\mathcal{M}$ can be relaxed by the constraint (II.8) since the Lagrangian is invariant under the constant shift $\mathcal{M}$ to $\mathcal{M}+c I$. Due to this fact, some of $\mathcal{M}$ 's can be shifted to projection matrices, which is used in (II.12)
} 
Hamiltonian of the system is

$$
\begin{array}{r}
H=\int d^{2} x \operatorname{tr}\left[\Pi \bar{\Pi}-\left|\partial_{i} \phi\right|^{2}+\frac{1}{4}\left|\partial_{i} \bar{\phi} \phi-\bar{\phi} \partial_{i} \phi\right|^{2}+\frac{i}{2}\left(\partial_{i} \bar{\psi} \gamma^{i} \psi-\bar{\psi} \gamma^{i} \partial_{i} \psi\right)-\frac{i}{2}\left(\bar{\psi} \gamma^{i} \psi\right)\left(\partial_{i} \bar{\phi} \phi-\bar{\phi} \partial_{i} \phi\right)\right. \\
\left.+m^{2}\left\{(\bar{\phi} \mathcal{P} \phi)-(\bar{\phi} \mathcal{P} \phi)^{2}\right\}+m(\bar{\phi} \mathcal{P} \phi)(\bar{\psi} \psi)-m(\bar{\psi} \mathcal{P} \psi)+\frac{1}{4}\left(\bar{\psi} \gamma_{i} \psi\right)\left(\bar{\psi} \gamma^{i} \psi\right)-\frac{1}{4}(\bar{\psi} \psi)^{2}\right]
\end{array}
$$

where the conjugate momenta are given by

$$
\Pi_{a}^{i}=\frac{\delta}{\delta \dot{\phi}_{i}{ }^{a}} \int d^{2} x \mathcal{L}={\overline{\left(D_{0} \phi\right)}}_{a}^{i}, \quad \bar{\Pi}_{i}{ }^{a}=\frac{\delta}{\delta \dot{\bar{\phi}}_{a}^{i}} \int d^{2} x \mathcal{L}=\left(D_{0} \phi\right)_{i}{ }^{a} .
$$

We use Poisson brackets defined as follows

$$
\begin{aligned}
& \left\{\phi_{i}{ }^{a}(x), \Pi_{b}{ }^{j}(y)\right\}_{\text {P.B. }}=\delta_{b}{ }^{a} \delta_{i}{ }^{j} \delta(x-y), \\
& \left\{\bar{\phi}_{a}^{i}(x), \bar{\Pi}_{j}^{b}(y)\right\}_{\mathrm{P. \textrm {B } .}}=\delta_{a}{ }^{b} \delta_{j}{ }^{i} \delta(x-y), \\
& \left\{\psi_{\alpha i}^{a}(x), \psi_{b}^{\dagger \beta}{ }_{b}^{j}(y)\right\}_{\text {P.B. }}=-i \delta_{\alpha}^{\beta} \delta_{b}{ }^{a} \delta_{i}{ }^{j} \delta(x-y) .
\end{aligned}
$$

There are one Gauss law constraint

$$
\bar{\phi} \bar{\Pi}-\Pi \phi-i \bar{\psi} \gamma^{0} \psi=0
$$

and four second class constraints

$$
\begin{array}{ll}
C_{a}^{1 b}=\bar{\phi}_{a}{ }^{i} \phi_{i}{ }^{b}-\delta_{a}^{b} \approx 0, & C_{a}^{2}{ }_{a}^{b}=\Pi_{a}{ }^{i} \phi_{i}{ }^{b}+\bar{\phi}_{a}^{i} \bar{\Pi}_{i}{ }^{b} \approx 0, \\
C_{a}^{3 b}=\psi_{a}^{\dagger}{ }_{a}^{i} \phi_{i}{ }^{b} \approx 0, & C^{4}{ }_{a}^{b}=\bar{\phi}_{a}^{i} \psi_{i}{ }^{b} \approx 0 .
\end{array}
$$

We label the second class constraints as $C_{A} \equiv\left(C_{a}^{1}{ }_{a}^{b}, C_{b}^{2 a}, C_{b}^{3 a}, C^{4}{ }_{a}^{b}\right)\left(A=1,2, \ldots, 4 M^{2}\right)$, and then the Dirac matrix is given by

$$
\Omega=\left\{C_{A}, C_{B}\right\}_{\text {Р.в. }}=\left[\begin{array}{cccc}
0 & X & 0 & 0 \\
-X^{\mathrm{T}} & Y & 0 & 0 \\
0 & 0 & 0 & Z \\
0 & 0 & Z^{\mathrm{T}} & 0
\end{array}\right], \quad \Omega^{-1}=\left[\begin{array}{cccc}
X^{\mathrm{T}-1} Y X^{-1} & X & 0 & 0 \\
-X^{\mathrm{T}-1} & 0 & 0 & 0 \\
0 & 0 & 0 & Z^{\mathrm{T}-1} \\
0 & 0 & Z^{-1} & 0
\end{array}\right]
$$

where

$$
\begin{aligned}
& X_{A B} \equiv X_{a}^{b} ;_{c}{ }^{d}=\left\{C^{1}{ }_{a}^{b}, C^{2}{ }_{c}^{d}\right\}_{\text {Р.в. }}=2 \delta_{a}^{d} \delta_{c}^{b}, \\
& Y_{A B} \equiv Y_{a}^{b} ;_{c}{ }^{d}=\left\{C^{2}{ }_{a}^{b}, C^{2}{ }_{c}^{d}\right\}_{\text {р.в. }}=\delta_{a}{ }^{d}(\Pi \phi-\bar{\phi} \bar{\Pi})_{c}^{b}-\delta_{c}^{b}(\Pi \phi-\bar{\phi} \bar{\Pi})_{a}{ }^{d} \text {, } \\
& Z_{A B} \equiv Z_{a}^{b} ;_{c}^{d}=\left\{C^{3}{ }_{a}^{b}, C^{4}{ }_{c}^{d}\right\}_{\text {P.в. }}=-i \delta_{a}^{d} \delta_{c}^{d} \text {. }
\end{aligned}
$$

The Dirac bracket is defined by

$$
\left\{P_{A}(x), Q_{B}(y)\right\}_{\mathrm{D}}=\left\{P_{A}(x), Q_{B}(y)\right\}_{\text {Р.в. }}-\int d z d z^{\prime}\left\{P_{A}(x), C_{E}(z)\right\}_{\text {Р.в. }} \Omega^{-1 E F}\left(z, z^{\prime}\right)\left\{C_{F}\left(z^{\prime}\right), Q_{B}(y)\right\}_{\text {Р.в. }} .
$$

Then the Dirac brackets between the physical variables are given by

$$
\begin{aligned}
& \left\{\phi_{i}{ }^{a}(x), \phi_{j}{ }^{b}(y)\right\}_{\mathrm{D}}=0, \\
& \left\{\bar{\phi}_{a}^{i}(x), \bar{\phi}_{b}{ }^{j}(y)\right\}_{\mathrm{D}}=0 \text {, } \\
& \left\{\phi_{i}{ }^{a}(x), \bar{\phi}_{b}{ }^{j}(y)\right\}_{\mathrm{D}}=0 \text {, } \\
& \left\{\phi_{i}{ }^{a}(x), \Pi_{b}{ }^{j}(y)\right\}_{\mathrm{D}}=\delta_{b}{ }^{a}\left(\delta_{i}{ }^{j}-\frac{1}{2}{\phi_{i}}^{c} \bar{\phi}_{c}^{j}\right) \delta(x-y), \\
& \left\{\bar{\phi}_{a}{ }^{i}(x), \bar{\Pi}_{j}^{b}(y)\right\}_{\mathrm{D}}=\delta_{a}{ }^{b}\left(\delta_{j}{ }^{i}-\frac{1}{2} \phi_{j}{ }^{c} \bar{\phi}_{c}{ }^{i}\right) \delta(x-y), \\
& \left\{\phi_{i}{ }^{a}(x), \bar{\Pi}_{j}^{b}(y)\right\}_{\mathrm{D}}=-\frac{1}{2}{\phi_{i}}^{b} \phi_{j}{ }^{a} \delta(x-y), \\
& \left\{\bar{\phi}_{a}^{i}(x), \Pi_{b}^{j}(y)\right\}_{\mathrm{D}}=-\frac{1}{2} \bar{\phi}_{b}^{i} \bar{\phi}_{a}^{j} \delta(x-y),
\end{aligned}
$$




$$
\begin{aligned}
& \left\{\Pi_{a}^{i}(x), \Pi_{b}^{j}(y)\right\}_{\mathrm{D}}=\left[\frac{1}{4} \bar{\phi}_{b}{ }^{i} \bar{\phi}_{c}^{j}(\Pi \phi-\bar{\phi} \bar{\Pi})_{a}{ }^{c}-\frac{1}{4} \bar{\phi}_{c}{ }_{c} \bar{\phi}_{a}^{j}(\Pi \phi-\bar{\phi} \bar{\Pi})_{b}{ }^{c}-\frac{1}{2}\left(\bar{\phi}_{b}{ }^{i} \Pi_{a}^{j}-\Pi_{b}{ }^{i} \bar{\phi}_{a}^{j}\right)\right] \delta(x-y), \\
& \left\{\bar{\Pi}_{i}{ }^{a}(x), \bar{\Pi}_{j}^{b}(y)\right\}_{\mathrm{D}}=\left[\frac{1}{4} \phi_{i}{ }^{c} \phi_{j}{ }^{a}(\Pi \phi-\bar{\phi} \bar{\Pi})_{c}{ }^{b}-\frac{1}{4}{\phi_{i}}^{b} \phi_{j}{ }^{c}(\Pi \phi-\bar{\phi} \bar{\Pi})_{c}{ }^{a}-\frac{1}{2}\left(\phi_{i}{ }^{b} \bar{\Pi}_{j}{ }^{a}-\bar{\Pi}_{i}{ }^{b} \phi_{j}{ }^{a}\right)\right] \delta(x-y), \\
& \left\{\Pi_{a}^{i}(x), \bar{\Pi}_{j}^{b}(y)\right\}_{\mathrm{D}}=\left[\frac{1}{4} \bar{\phi}_{c}{ }^{i} \phi_{j}{ }^{c}(\Pi \phi-\bar{\phi} \bar{\Pi})_{a}^{b}-\frac{1}{4} \bar{\phi}_{c}{ }^{i} \phi_{j}{ }^{d}(\Pi \phi-\bar{\phi} \bar{\Pi})_{d}^{c} \delta_{a}^{b}-\frac{1}{2}\left(\bar{\phi}_{c}{ }^{i} \bar{\Pi}_{j}{ }^{c}-\Pi_{c}{ }^{i} \phi_{j}{ }^{c}\right) \delta_{a}{ }^{b}\right. \\
& \left.+i \psi_{c}^{\dagger}{ }_{c}^{i} \psi_{j}^{c} \delta_{a}^{b}\right] \delta(x-y) \text {, } \\
& \left\{\Pi_{a}^{i}(x), \psi_{b}^{\dagger \alpha}{ }^{j}(y)\right\}_{\mathrm{D}}=\psi_{b}^{\dagger \alpha}{ }_{b}^{i} \bar{\phi}_{a}^{j} \delta(x-y), \\
& \left\{\bar{\Pi}_{i}{ }^{a}(x), \psi_{\alpha j}{ }^{b}(y)\right\}_{\mathrm{D}}=\psi_{\alpha i}{ }^{b} \phi_{j}{ }^{a} \delta(x-y), \\
& \left\{\psi_{\alpha i}^{a}(x), \psi_{b}^{\dagger \beta}{ }_{b}^{j}(y)\right\}_{\mathrm{D}}=-i \delta_{\alpha}^{\beta} \delta_{b}{ }^{a}\left(\delta_{i}{ }^{j}-{\phi_{i}}^{c} \bar{\phi}_{c}^{j}\right) \delta(x-y) .
\end{aligned}
$$

We use Noether procedure to obtain the various conserved charges. First the supercharge is

$$
Q_{\alpha}=\int d^{2} x \operatorname{tr}\left[\Pi \psi_{\alpha}+\partial_{i} \bar{\phi}\left(\gamma^{i} \gamma^{0} \psi\right)_{\alpha}-i m \bar{\phi} \mathcal{P}\left(\gamma^{0} \psi\right)_{\alpha}\right] .
$$

The Hamiltonian is given by (III.1) and the momenta

$$
P^{i}=\int d^{2} x \operatorname{tr}\left[\Pi \partial^{i} \phi+\partial^{i} \bar{\phi} \bar{\Pi}+\frac{i}{2}\left(\bar{\psi} \gamma^{0} \partial^{i} \psi-\partial^{i} \bar{\psi} \gamma^{0} \psi\right)+\frac{1}{2}\left(-i \Pi \phi+i \bar{\phi} \bar{\Pi}+\bar{\psi} \gamma^{0} \psi\right)\left(i \partial^{i} \bar{\phi} \phi-i \bar{\phi} \partial^{i} \phi-\bar{\psi} \gamma^{i} \psi\right)\right],
$$

where the last term in (III.23) is a gauge degree of freedom. There is also a symmetry under the transformations $\delta \phi=i \mathcal{P} \phi$ and $\delta \psi=i \mathcal{P} \psi$, and the corresponding scalar Noether charge is

$$
U=\int d^{2} x \operatorname{tr}\left[i \Pi \mathcal{P} \phi-i \bar{\phi} \mathcal{P} \bar{\Pi}-\bar{\psi} \mathcal{P} \gamma^{0} \psi\right]
$$

We compute explicitly to obtain the Dirac brackets among the supercharges using the relations (III.12)-(III.21)

$$
\left\{Q_{\alpha}, Q^{\dagger \beta}\right\}_{\mathrm{D}}=-i\left(\gamma^{\mu} \gamma^{0}\right)_{\alpha}^{\beta} P_{\mu}-i m \gamma_{\alpha}^{0}{ }_{\alpha}^{\beta} U-i \gamma_{\alpha}^{0}{ }_{\alpha}^{\beta}(2 \pi T)+\gamma_{\alpha}^{i{ }_{\alpha} R_{i}},
$$

where

$$
\begin{aligned}
T & =\frac{i}{2 \pi} \int d^{2} x \operatorname{tr}\left[\epsilon^{i j}\left\{\left(\partial_{i} \bar{\phi}\right)\left(\partial_{j} \phi\right)+\frac{i}{2} \partial_{i}\left(\bar{\psi} \gamma_{j} \psi\right)\right\}\right] \\
R_{i} & =\int d^{2} x \operatorname{tr}\left[\frac{1}{2} \partial_{i}(\bar{\psi} \psi)+m \partial_{i}(\bar{\phi} \mathcal{P} \phi)\right] .
\end{aligned}
$$

\section{QUANTIZATION OF DIRAC BRACKETS}

We quantize Dirac brackets (III.12)-(III.21). Assuming that the ordering of the second class constraints are fixed as in (III.5), one of the possible choices of the ordering which makes all the dynamical variables commute with the second class constraints is given by

$$
\begin{aligned}
{\left[\phi_{i}{ }^{a}(x), \Pi_{b}{ }^{j}(y)\right] } & =i \delta_{b}{ }^{a}\left(\delta_{i}{ }^{j}-\frac{1}{2}{\phi_{i}}^{c} \bar{\phi}_{c}^{j}\right) \delta(x-y), \\
{\left[\bar{\phi}_{a}{ }^{i}(x), \bar{\Pi}_{j}{ }^{b}(y)\right] } & =i \delta_{a}{ }^{b}\left(\delta_{j}{ }^{i}-\frac{1}{2} \phi_{j}{ }^{c} \bar{\phi}_{c}^{i}\right) \delta(x-y), \\
{\left[\phi_{i}{ }^{a}(x), \bar{\Pi}_{j}{ }^{b}(y)\right] } & =-\frac{i}{2}{\phi_{i}}^{b} \phi_{j}{ }^{a} \delta(x-y), \\
{\left[\bar{\phi}_{a}{ }^{i}(x), \Pi_{b}{ }^{j}(y)\right] } & =-\frac{i}{2} \bar{\phi}_{b}{ }^{i} \bar{\phi}_{a}^{j} \delta(x-y), \\
{\left[\Pi_{a}{ }^{i}(x), \Pi_{b}{ }^{j}(y)\right] } & =\left[\frac{i}{4}(\Pi \phi-\bar{\phi} \bar{\Pi})_{a}^{c} \bar{\phi}_{b}{ }^{i} \bar{\phi}_{c}^{j}-\frac{i}{4}(\Pi \phi-\bar{\phi} \bar{\Pi})_{b}{ }^{c} \bar{\phi}_{c}^{i} \bar{\phi}_{a}^{j}-\frac{i}{2}\left(\bar{\phi}_{b}{ }^{i} \Pi_{a}^{j}-\bar{\phi}_{a}^{j} \Pi_{b}{ }^{i}\right)\right] \delta(x-y), \\
{\left[\bar{\Pi}_{i}{ }^{a}(x), \bar{\Pi}_{j}{ }^{b}(y)\right] } & =\left[\frac{i}{4} \phi_{i}{ }^{c} \phi_{j}{ }^{a}(\Pi \phi-\bar{\phi} \bar{\Pi})_{c}{ }^{b}-\frac{i}{4} \phi_{i}{ }^{b} \phi_{j}{ }^{c}(\Pi \phi-\bar{\phi} \bar{\Pi})_{c}{ }^{a}-\frac{i}{2}\left(\bar{\Pi}_{j}{ }^{a} \phi_{i}{ }^{b}-\bar{\Pi}_{i}{ }^{b} \phi_{j}{ }^{a}\right)\right] \delta(x-y),
\end{aligned}
$$




$$
\begin{aligned}
& {\left[\Pi_{a}^{i}(x), \bar{\Pi}_{j}^{b}(y)\right]=\left[\frac{i}{4} \bar{\phi}_{c}{ }^{i} \phi_{j}{ }^{c}(\Pi \phi-\bar{\phi} \bar{\Pi})_{a}^{b}-\frac{i}{4} \bar{\phi}_{c}{ }^{i} \phi_{j}{ }^{d}(\Pi \phi-\bar{\phi} \bar{\Pi})_{d}^{c} \delta_{a}^{b}-\frac{i}{2}\left(\bar{\phi}_{c}{ }^{i} \bar{\Pi}_{j}{ }^{c}-\phi_{j}{ }^{c} \Pi_{c}{ }^{i}\right) \delta_{a}^{b}\right.} \\
& \left.-\psi_{c}^{\dagger}{ }_{c}^{i} \psi_{j}^{c} \delta_{a}^{b}-h\left\{\psi_{j}^{c}, \psi_{c}^{\dagger}{ }_{c}^{i}\right\} \delta_{a}^{b}\right] \delta(x-y), \\
& {\left[\Pi_{a}^{i}(x), \psi_{b}^{\dagger \alpha}{ }^{j}(y)\right]=i \psi_{b}^{\dagger \alpha}{ }_{b}^{i} \bar{\phi}_{a}^{j} \delta(x-y),} \\
& {\left[\bar{\Pi}_{i}{ }^{a}(x), \psi_{\alpha j}{ }^{b}(y)\right]=i \psi_{\alpha i}{ }^{b} \phi_{j}{ }^{a} \delta(x-y),} \\
& \left\{\psi_{\alpha i}^{a}(x), \psi_{b}^{\dagger \beta}{ }_{b}^{j}(y)\right\}=\delta_{\alpha}^{\beta} \delta_{b}^{a}\left(\delta_{i}^{j}-\phi_{i}^{c} \bar{\phi}_{c}^{j}\right) \delta(x-y) \text {. }
\end{aligned}
$$

Note that with the above choice, (IV.5) and (IV.6) vanish for identical indices. This is the same as the method of [33], where the Dirac analysis is used for bosonic $\mathrm{CP}^{N}$ model. In the above (IV.7) the ordering parameter $h$ is undetermined. It will be fixed by the SUSY algebra.

Since the supercharge in (III.22) does not have any ordering ambiguity, a straightforward computation yields the following quantum SUSY algebra.

$$
\begin{aligned}
\left\{Q_{\alpha}, Q^{\dagger \beta}\right\}= & \delta_{\alpha}^{\beta} \int d^{2} x \operatorname{tr}\left[\Pi \bar{\Pi}-\left|\partial_{i} \bar{\phi}\right|^{2}+\frac{1}{4}\left|\partial_{i} \bar{\phi} \phi-\bar{\phi} \partial_{i} \phi\right|^{2}+\frac{i}{2}\left(\partial_{i} \bar{\psi} \gamma^{i} \psi-\bar{\psi} \gamma^{i} \partial_{i} \psi\right)-\frac{i}{2}\left(\bar{\psi} \gamma^{i} \psi\right)\left(\partial_{i} \bar{\phi} \phi-\bar{\phi} \partial_{i} \phi\right)\right. \\
& \left.+m^{2}\left\{(\bar{\phi} \mathcal{P} \phi)-(\bar{\phi} \mathcal{P} \phi)^{2}\right\}+m(\bar{\phi} \mathcal{P} \phi)(\bar{\psi} \psi)-m(\bar{\psi} \mathcal{P} \psi)+\frac{1}{4}\left(\bar{\psi} \gamma_{i} \psi\right)\left(\bar{\psi} \gamma^{i} \psi\right)-\frac{1}{4}(\bar{\psi} \psi)^{2}\right] \\
& +\int d^{2} x \delta_{\alpha}^{\beta} \frac{1}{2} \delta_{a}^{a}\left(\bar{\psi} \gamma^{0} \psi\right)_{b}^{b}+\left(2 h+\frac{1}{2}\right) \delta_{a}{ }^{a}\left\{\left(\bar{\psi} \gamma^{0}\right)^{\beta} \psi_{\alpha}\right\}_{b}{ }^{b} \\
& +\left(\gamma^{i} \gamma^{0}\right)_{\alpha}^{\beta} \int d^{2} x \operatorname{tr}\left[\Pi \partial_{i} \phi+\partial_{i} \bar{\phi} \bar{\Pi}+\frac{i}{2}\left(\bar{\psi} \gamma^{0} \partial_{i} \psi-\partial_{i} \bar{\psi} \gamma^{0} \psi\right)\right] \\
& +m \gamma_{\alpha}^{0}{ }_{\alpha}^{\beta} \int d^{2} x \operatorname{tr}\left[i \Pi \mathcal{P} \phi-i \bar{\phi} \mathcal{P} \bar{\Pi}-\bar{\psi} \mathcal{P} \gamma^{0} \psi\right] \\
& +\gamma_{\alpha}^{0}{ }_{\alpha}^{\beta}(2 \pi) \int d^{2} x \operatorname{tr}\left[\frac{i \epsilon^{i j}}{2 \pi}\left\{\left(\partial_{i} \bar{\phi}\right)\left(\partial_{j} \phi\right)+\frac{i}{2} \partial_{i}\left(\bar{\psi} \gamma_{j} \psi\right)\right\}\right] \\
& +i \gamma_{\alpha}^{i \beta} \int d^{2} x \operatorname{tr}\left[\frac{1}{2} \partial_{i}(\bar{\psi} \psi)+m \partial_{i}(\bar{\phi} \mathcal{P} \phi)\right] .
\end{aligned}
$$

The first term in the third line arises from the ordering of the last two quartic terms of $\psi$ in (III.1), and therefore it can be absorbed in the definition of energy. We may appropriately fix the parameter $h$ to eliminate the second term in the line to make sure that the SUSY algebra closes at quantum level. We choose $h=-\frac{1}{4}$ to get quantum SUSY algebra of the form,

$$
\left\{Q_{\alpha}, Q^{\dagger \beta}\right\}=\left(\gamma^{\mu} \gamma^{0}\right)_{\alpha}^{\beta} P_{\mu}+m \gamma_{\alpha}^{0} U+\gamma_{\alpha}^{0}(2 \pi T)+i \gamma_{\alpha}^{i} R_{i}
$$

where the quantum Hamiltonian is given by

$$
\begin{aligned}
P^{0}=\int & d^{2} x \operatorname{tr}\left[\Pi \bar{\Pi}-\left|\partial_{i} \bar{\phi}\right|^{2}+\frac{1}{4}\left|\partial_{i} \bar{\phi} \phi-\bar{\phi} \partial_{i} \phi\right|^{2}+\frac{i}{2}\left(\partial_{i} \bar{\psi} \gamma^{i} \psi-\bar{\psi} \gamma^{i} \partial_{i} \psi\right)-\frac{i}{2}\left(\bar{\psi} \gamma^{i} \psi\right)\left(\partial_{i} \bar{\phi} \phi-\bar{\phi} \partial_{i} \phi\right)\right. \\
& \left.+m^{2}\left\{(\bar{\phi} \mathcal{P} \phi)-(\bar{\phi} \mathcal{P} \phi)^{2}\right\}+m(\bar{\phi} \mathcal{P} \phi)(\bar{\psi} \psi)-m(\bar{\psi} \mathcal{P} \psi)+\frac{1}{4}\left(\bar{\psi} \gamma_{i} \psi\right)\left(\bar{\psi} \gamma^{i} \psi\right)-\frac{1}{4}(\bar{\psi} \psi)^{2}+\frac{1}{2} M\left(\bar{\psi} \gamma^{0} \psi\right)\right]
\end{aligned}
$$

Here $M$ is the number of color indices and the other operators are the same as (III.23), (III.24), (III.26) and (III.27).

It can be shown that the SUSY algebra (IV.12) can be rewritten as

$$
\begin{aligned}
& \left\{Q_{ \pm \alpha}, Q_{ \pm}^{\dagger \beta}\right\}=\frac{1}{4}\left[\left\{\gamma^{0}, \gamma^{\mu}\right\}_{\alpha}^{\beta} \pm\left(\gamma^{\mu}+\gamma^{\mu \dagger}\right)_{\alpha}^{\beta}\right] P_{\mu}+\frac{1}{2} m\left(\gamma_{\alpha}^{0}{ }_{\alpha}^{\beta} \pm \delta_{\alpha}^{\beta}\right) U+\frac{1}{2}\left(\gamma_{\alpha}^{0}{ }_{\alpha}^{\beta} \pm \delta_{\alpha}^{\beta}\right) 2 \pi T, \\
& \left\{Q_{ \pm \alpha}, Q_{ \pm}^{\dagger \alpha}\right\}=P_{0} \pm m U \pm 2 \pi T, \\
& \left\{Q_{ \pm \alpha}, Q_{\mp}^{\dagger \beta}\right\}=\frac{1}{4}\left\{\left[\gamma^{\mu}, \gamma^{0}\right]_{\alpha}^{\beta} \mp\left(\gamma^{\mu}-\gamma^{\mu \dagger}\right)_{\alpha}^{\beta}\right\} P_{\mu}+\frac{i}{2}\left(\gamma_{\alpha}^{i \beta} \pm\left(\gamma^{0} \gamma^{i}\right)_{\alpha}^{\beta}\right) R_{i}, \\
& \left\{Q_{ \pm \alpha}, Q_{\mp}^{\dagger \alpha}\right\}=0,
\end{aligned}
$$


where we redefine supercharges as $Q_{ \pm \alpha} \equiv\left(\frac{1 \pm \gamma^{0}}{2} Q\right)_{\alpha}$. The explicit forms of the $Q_{ \pm}$and $Q_{ \pm}^{\dagger}$ are

$$
\begin{aligned}
Q_{ \pm} & =\frac{1}{2} \int d^{2} x \operatorname{tr}\left[\left(1 \pm \gamma^{0}\right)\left(\partial_{0} \bar{\phi} \mp i m \bar{\phi} \mathcal{P}\right) \psi+\left(\gamma^{i} \gamma^{0} \mp \gamma^{i}\right) \partial_{i} \bar{\phi} \psi\right] \\
Q_{ \pm}^{\dagger} & =\frac{1}{2} \int d^{2} x \operatorname{tr}\left[\psi^{\dagger}\left(1 \pm \gamma^{0}\right)\left(\partial_{0} \phi \pm i m \mathcal{P} \phi\right)+\psi^{\dagger}\left(\gamma^{i} \gamma^{0} \pm \gamma^{i}\right) \partial_{i} \phi\right]
\end{aligned}
$$

From (IV.15), the energy is bounded as $P_{0} \geq m|U|+2 \pi|T|$ and the saturation occurs when $Q_{+}=0$ or $Q_{-}=0$, i.e. the following Bogomolnyi equations are satisfied

$$
\begin{aligned}
\partial_{0} \phi \pm i m \mathcal{P} \phi & =0, \\
\partial_{i} \phi \mp i \epsilon_{i j} \partial^{j} \phi & =0, \quad\left(\epsilon_{12}=1\right),
\end{aligned}
$$

which shows that the $Q$-lumps are $\frac{1}{2}$ BPS objects $[9]$. With these BPS equations satisfied, the energy is given by

$$
P_{0}=m|U|+2 \pi|T| .
$$

\section{CONCLUSION}

In this paper, we studied $\mathcal{N}=2$ massive Grassmannian sigma model in $2+1$ dimensions. We derived the off-shell action by Scherk-Schwarz dimensional reduction from $\mathcal{N}=1$ formalism in $3+1$ dimensions. We performed canonical analysis via Dirac method and computed SUSY algebra. The SUSY algebra with central charge extension was obtained with a fixed choice of the operator ordering.

It would be interesting to check whether other choices of ordering yield the same SUSY algebra and to extend the present formalism to $\mathcal{N}=4$ Grassmannian model in $2+1$ dimensions.

\section{Acknowledgments}

We thank Jeong-Hyuck Park for early participation. We are also grateful to Masato Arai, Dongsu Bak and Rabin Banerjee for useful discussions. The work of PO and SS is supported by the Science Research Center Program of the Korea Science and Engineering Foundation through the Center for Quantum Spacetime(CQUeST) of Sogang University with grant No. R11-2005-021 and by Korea Research Foundation Grant (R05-2004-000-10682-0). The work of $\mathrm{HN}$ is the result of research activities (Astrophysical Research Center for the Structure and Evolution of the Cosmos (ARCSEC)) and grant No. R01-2006-000-10965-0 from the Basic Research Program supported by KOSEF.

[1] R. A. Leese, Nucl. Phys. B 366, 283 (1991)

[2] E. Abraham, Phys. Lett. B 278, 291 (1992); E. Abraham, P. K. Townsend, Phys. Lett. B 291, 85 (1992); E. Abraham, P. K. Townsend, Phys. Lett. B 295, 225 (1992)

[3] S. Coleman, Nucl. Phys. B 262, 263 (1985).

[4] J. P. Gauntlett, R. Portugues, P. K. Townsend, Phys. Rev. D 63, 085002(2001) hep-th/0008221.

[5] J. P. Gauntlett, D. Tong, P. K. Townsend, Phys. Rev. D64, 025010 (2001) hep-th/0012178.

[6] M. Naganuma, M. Nitta, N. Sakai, Grav. Cosmol. 8, 129 (2002) hep-th/0108133.

[7] Y. Isozumi, M. Nitta, K. Ohashi, N. Sakai, Phys. Rev. D 71, 065018 (2005) hep-th/0405129.

[8] R. S. Ward, J. Math. Phys. 44, 3555 (2003) hep-th/0302045.

[9] D. Bak, S. O. Hahn, J. Lee and P. Oh, Phys. Rev. D 75, 025004 (2007) hep-th/0610067.

[10] R. Portugues, P. K. Townsend, JHEP 0204, 039 (2002) hep-th/0203181.

[11] P. K. Townsend, Comptes Rendus Physique 6, 271 (2005) hep-th/0411206].

[12] D. Mateos, P. K. Townsend, Phys. Rev. Lett. 87, 011602 (2001) hep-th/0103030].

[13] P. K. Townsend, Annales Henri Poincare 4, S183 (2003) hep-th/0211008.

[14] D. Tong (2005) hep-th/0509216.

[15] M. Eto, Y. Isozumi, M. Nitta, K. Ohashi, N. Sakai, J. Phys. A 39, R315 (2006) hep-th/0602170.

[16] M. Shifman, A. Yung, Rev. Mod. Phys. 79, 1139 (2007) hep-th/0703267.

[17] M. Eto, Y. Isozumi, M. Nitta, K. Ohashi, Nucl. Phys. B 752, 140 (2006) hep-th/0506257.

[18] E. Witten, D. Olive, Phys. Lett. B 78, 97 (1978).

[19] See also Z. Hlousek, D. Spector, Nucl. Phys. B 370, 143 (1992); S. Ferrara, M. Porrati, Phys. Lett. B 423,255 (1998). 
[20] S. Aoyama, Nucl. Phys. B 168, 354 (1980).

[21] S. Rouhani, Nucl. Phys. B 169, 430 (1980).

[22] J. Scherk, J. H. Schwarz, Nucl. Phys. B 153, 61 (1979).

[23] J. Wess, J Bagger, Supersymmetry and Supergravity, Princeton University Press (1992).

[24] L. Alvarez-Gaume, D. Z. Freedman, Commun. Math. Phys. 91, 87 (1983).

[25] S.James Gates, Jr., Nucl. Phys. B 238, 349 (1984).

[26] A. C. Davis, M. D. Freeman, A. J. Macfarlane, Nucl. Phys. B 256, 299 (1985).

[27] D. Tong, Phys. Rev. D 66, 025013 (2002) hep-th/0202012.

[28] M. Arai, M. Naganuma, M. Nitta, N. Sakai, Nucl. Phys. B 652, 35 (2003) hep-th/0211103.

[29] M. Arai, M. Nitta, N. Sakai, Prog. Theor. Phys. 113, 657 (2005) hep-th/0307274.

[30] A. Losev, M. Shifman, Phys. Rev. D 68, 045006 (2003) hep-th/0304003.

[31] A. Gorsky, M. Shifman, A. Yung, Phys. Rev. D 73, 065011 (2006) hep-th/0512153.

[32] M. Shifman, A. Vainshtein, R. Zwicky J. Phys. A 39, 13005 (2006) hep-th/0602004.

[33] C. G. Han, Phys. Rev. D 47, 5521 (1993). 